\title{
EL PLAN E COMO ESTÍMULO FISCAL: EVALUACIÓN DE SU EFECTIVIDAD EN LA CREACIÓN Y MANTENIMIENTO DE EMPLEO A ESCALA PROVINCIAL
}

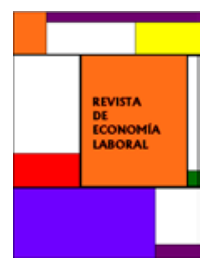

\author{
André Carrascal Incera ${ }^{1}$, Fernando Corbelle Cacabelos, \\ Melchor Fernández, $\mathrm{M}^{\mathrm{a}}$ Carmen Vilariño López \\ Grupo de Análise e Modelización Económica (GAME) \\ Instituto Universitario de Estudos e Desenvolvemento de Galicia (IDEGA) \\ Universidade de Santiago de Compostela
}

Recibido 2 Diciembre 2013; aceptado 27 Mayo 2014

\section{Resumen}

En el actual contexto económico, cobran especial relevancia la preocupación por el estímulo y la recuperación del empleo, así como el análisis de la eficacia de las medidas diseñadas con este objetivo. El objetivo principal del presente trabajo es evaluar los efectos del Fondo Estatal de Inversión Local (principal medida de expansión de la demanda dentro del Plan E) sobre el empleo una vez finalizada su ejecución. Además, se analiza si el reparto homogéneo de los fondos en términos per cápita, ha ofrecido unos resultados también homogéneos en cuanto al estímulo laboral logrado a escala provincial. Finalmente, y dado que el estudio confirma la heterogeneidad de los resultados, se realiza una aproximación a las posibles causas de dicha disparidad.

Palabras clave: Plan E, Ratio de Ajuste Territorial del Mercado Laboral, Paro Registrado. Clasificación JEL: H30, H54, J68, R23.

\section{Abstract}

In the current economic context, the concern about employment and its recovery is highly important. Therefore, we must pay special attention to the analysis and the strategies designed for this purpose. The main aim of this paper is to evaluate the effects of the Local Investment Fund (main policy to expand demand included on Plan E) on employment in the Spanish provinces. Moreover, it is also examined if the homogeneous share in terms of funds per capita has presented homogeneous labour results at provincial level. Finally, since this study confirms the heterogeneity of the results, an approach of the possible causes of this disparity is made.

Key words: Plan E, ratio of geographical labour market adjustment, registered unemployment.

JEL Classification: H30, H54, J68, R23.

${ }^{1}$ Autor para correspondencia:_andre.carrascal@rai.usc.es.

(C) Revista de Economía Laboral 


\section{Introducción}

La crisis financiera internacional iniciada a finales del verano de 2007 se trasladó rápidamente a las economías europeas con importantes repercusiones en la evolución de la producción y el empleo en los años siguientes. De acuerdo con las estimaciones oficiales ${ }^{2}$, para el conjunto de la economía española el crecimiento del PIB pasó del 3,5\% en 2007, al 0,9\% en 2008 y al -3,8\% en 2009. La evolución del empleo (medido en puestos de trabajo equivalentes) fue mucho más negativa pasando de un crecimiento del $3,0 \%$ en 2007 al $-0,2 \%$ en 2008 y al $-6,2 \%$ en 2009 . Esta evolución negativa tan brusca del mercado laboral, elevó la tasa de paro hasta el 20,5\% en 2010 confirmando a España como el líder del desempleo en la Unión Europea (duplicando la tasa de paro en la zona euro). No obstante, a escala territorial, los efectos de la crisis sobre el empleo no se distribuyeron homogéneamente. De hecho, las diferencias en la destrucción de empleo a escala provincial han sido muy importantes. En los dos primeros años de crisis la tasa de paro aumentó entre los 2,3 puntos porcentuales de Segovia y los 11,5 puntos en Córdoba.

En este contexto general de destrucción fuerte de empleo el gobierno español, al igual que las autoridades económicas de todo el mundo, adoptó distintas medidas de política económica entre las que destacan las destinadas a expandir la demanda a corto plazo que se englobaron bajo la denominación de "Plan español para el Estímulo de la Economía y el Empleo", también conocido como Plan E. Por el lado de los gastos, las dos medidas fundamentales adoptadas en 2009 con el objetivo de paliar los efectos de la crisis económica fueron la creación del Fondo Estatal de Inversión Local (FEIL) y del Fondo Especial del Estado para la Dinamización de la Economía y el Empleo (ambas adoptadas en noviembre de 2008).

El FEIL, gestionado inicialmente por el Ministerio de Administraciones Públicas, estaba dotado con 8.000 millones de euros (0,75\% del PIB) y tenía como objetivo principal incrementar la inversión pública en el ámbito local mediante la financiación de obras públicas de nueva planificación y ejecución inmediata, que fuesen competencia de las propias entidades locales. Se trataba de un fondo de carácter extraordinario con el que se pretendía primordialmente generar y mantener empleo, sobre

2 INE, Contabilidad nacional de España. Base 2008, serie contable 2000-2012. www.ine.es. 
todo en el sector de la construcción y en las industrias vinculadas al mismo, dado el importante ajuste al que se estaban viendo sometidos con motivo de la crisis económica. Como se afirma en el Informe de seguimiento del Fondo Estatal de Inversión Local (FEIL) presentado por el Ministerio de Política Territorial (MPT, 2010), ${ }^{3}$ el FEIL, al tiempo que trata de favorecer el empleo, también buscaba reforzar la capitalización de los municipios, en tanto que la financiación prevista en este fondo extraordinario estaba dirigida hacia proyectos que conllevaban mejoras en las dotaciones municipales de infraestructuras, tanto productivas como de utilidad social.

El balance general realizado desde el propio gobierno fue muy optimista, tanto respecto al efecto sobre el empleo, como sobre la capitalización de los municipios. Pero sobre todo, el FEIL habría logrado un éxito rotundo respecto al primer objetivo (mantener y crear empleo), lo que sirvió como principal argumento para el desarrollo durante 2010 del "Fondo Estatal para el Empleo y la Sostenibilidad Local”, que suponía, en cierto modo, una continuación del FEIL. Según el informe del MPT, las obras ejecutadas durante 2009 habrían dado empleo a 426.195 personas, de la cuales 181.335 son de nueva contratación y 244.860 ya estaban contratadas en las empresas adjudicatarias y mantienen, por tanto, su empleo. De este modo, se han superado los objetivos de empleo inicialmente previstos, que se situaban en el entorno de los 277.539 (entre nuevos empleos y mantenimiento de puestos de trabajo existentes). ${ }^{4}$

Dada la relevancia del impacto sobre el empleo del FEIL reconocida por el gobierno, parece que el debate académico (teórico y empírico) sobre el valor del multiplicador del gasto público, y en consecuencia de la validez de la política fiscal como estímulo de la economía en situaciones de insuficiencia de demanda parece finalmente cerrado (Uxó et al., 2010). No obstante, se mantienen ciertas dudas sobre el impacto real del FEIL ${ }^{5}$.

${ }^{3}$ Desde abril de 2009 corresponde al Ministerio de Política Territorial la gestión del FEIL, tras la reorganización del gobierno y la desaparición del Ministerio de Administraciones Públicas.

4 En un reciente informe del Tribunal de Cuentas se concluye que los 30.698 proyectos del FEIL generaron 201.195 contratados (cuadros incluidos en las páginas 111 y 113 del documento). Lejos por tanto, de lo inicialmente previsto en las previsiones comunicadas por las entidades locales en la solicitud de fondos (277.539), pero lejísimos de las 426.195 contrataciones que los proyectos adjudicados indicaban en sus propuestas.

5 Primero, sobre la magnitud del impacto agregado sobre el empleo. Debemos recordar que el análisis realizado desde el MPT se basa en las estimaciones sobre la creación de empleo a partir de la información incluida en los proyectos presentados por las empresas 
El objetivo de este artículo es evaluar los efectos agregados y territoriales del FEIL a nivel provincial una vez finalizado su plazo de ejecución. En primer lugar, se presenta el Fondo y sus principales magnitudes y diferencias provinciales (número de proyectos, cuantía total, empleos presentados, etc.). Así, centraremos el análisis descriptivo en variables obtenidas a escala provincial que nos parecen fundamentales para entender los resultados finales: la financiación por desempleado (obtenida del Fondo), el porcentaje de afiliados al sector de la construcción y la evolución del total de la inversión en infraestructuras públicas (licitación pública para la construcción de edificaciones y obra pública en la provincia realizada por la administración central y autonómica). En la segunda sección, aproximamos la situación del mercado laboral a escala provincial a partir del cálculo de lo que hemos denominado Ratio de Ajuste Territorial del Mercado Laboral (RATML). Con la finalidad de evaluar el criterio de reparto utilizado (homogéneo en términos per cápita) realizamos una regresión que testa de forma conjunta la relación entre las variables citadas y la situación del mercado laboral medido a partir de la ratio propuesta, antes y después de la ejecución del Fondo. Finalmente, en el último apartado se presentan las principales conclusiones que se pueden extraer de este artículo.

\section{El Fondo Estatal de Inversión Local}

El principal objeto del Fondo Estatal de Inversión Local (FEIL) es aumentar la financiación pública y contribuir a dinamizar las economías locales, favoreciendo la creación y mantenimiento de puestos de trabajo, además de incrementar el potencial inversor de los ayuntamientos. Por lo tanto, la evaluación de la consecución de los objetivos previstos debe

adjudicatarias. Por lo tanto, la estimación agregada del efecto se realiza desde una perspectiva microeconómica de equilibrio parcial que puede provocar una sobrestimación de la repercusión de la inversión propuesta al no considerar el contexto general en el que se realiza. Es decir, sabemos la respuesta en términos de empleo de las empresas que resultan adjudicatarias de los proyectos, pero desconocemos cual será la reacción de las empresas que no consiguen esa adjudicación. En la estimación realizada desde el MPT se está suponiendo que estas empresas no reaccionan en ninguna dirección (ceteris paribus). Segundo, existen también dudas sobre los efectos territoriales del FEIL, en especial sus efectos redistributivos. Un fondo de inversión repartido en términos per cápita puede provocar nuevos desequilibrios territoriales en producción y empleo, sobre todo si la efectividad de la inversión pública depende del nivel de la misma (efectos aglomeración y red). 
focalizarse en la evaluación de los resultados del mercado laboral, tanto a escala estatal como provincial.

La evolución del mercado laboral (Cuadro 1) ha sido muy negativa en los dos primeros años de la crisis económica, tanto medido en número de afiliados a la Seguridad Social como en la variación del empleo (en puestos de trabajo equivalentes), muy condicionada por la evolución del sector de la construcción. El importante esfuerzo realizado por el gobierno a través del FEIL no impidió la pérdida de aproximadamente 217.980 afiliados en la construcción durante el año 2009. Aun así, la situación mejoró sensiblemente respecto a lo acontecido en el año 2008 donde la pérdida de empleo en el sector más que doblo la cantidad anterior (560.000 afiliados).

\section{Cuadro 1: Evolución de los principales agregados macroeconómicos (en millones de euros) y variables del mercado laboral (en miles de personas)}

\begin{tabular}{lccccccc}
\hline & $\begin{array}{c}\text { Variación } \\
\text { relativa } \\
09 / 08\end{array}$ & $\begin{array}{c}\text { Variación } \\
\text { relativa } \\
08 / 07\end{array}$ & $\begin{array}{c}\text { Variación } \\
\text { absoluta } \\
09 / 08\end{array}$ & $\begin{array}{c}\text { Variación } \\
\text { absoluta } \\
08 / 07\end{array}$ & 2009 & 2008 & 2007 \\
\hline PIB & $-3,72 \%$ & $0,86 \%$ & -29.938 & 6.856 & 774.285 & 804.223 & 797.367 \\
$\begin{array}{l}\text { Formación Bruta } \\
\text { de Capital fijo }\end{array}$ & $-15,81 \%$ & $-4,29 \%$ & -35.994 & -10.201 & 191.657 & 227.651 & 237.852 \\
Empleo (PTE) & $-6,61 \%$ & $-0,53 \%$ & -1.256 & -101 & 17.733 & 18.988 & 19.090 \\
Afiliados totales * & $-4,31 \%$ & $-3,95 \%$ & -740 & -706 & 16.432 & 17.172 & 17.878 \\
Afiliados constr. * & $-12,19 \%$ & $-23,86 \%$ & -218 & -561 & 1.570 & 1.788 & 2.349 \\
& & & & & & &
\end{tabular}

* A 31 de diciembre.

Fuente: Elaboración propia a partir de datos del INE y la Tesorería general de la Seguridad Social.

En el Cuadro 2 se resumen las previsiones de las principales magnitudes del FEIL en España por provincias. El Ministerio de Administraciones Públicas en el desempeño de su labor de gestión de los fondos, decidió, en su momento, un reparto homogéneo de los mismos de acuerdo a la población total del municipio (una cantidad de 176,99 euros por habitante), lo que se comunicó como un ejercicio de objetividad y transparencia. Como existen importantes diferencias en la tasa de actividad y paro por provincias, una distribución homogénea de los fondos en términos per cápita genera importantes diferencias en su distribución 
por empleado o por desempleado, grupo este último sobre el que el Plan E debía incidir. ${ }^{6}$ Como se aprecia en el Gráfico 1, la proporción de personas paradas en las provincias es sensiblemente diferente, dando como resultado que la financiación efectiva por persona desempleada resulte ser claramente distinta. Evidentemente, este hecho puede distorsionar los efectos sobre la creación y el mantenimiento de las cifras de empleo (o reducción de las de desempleo) en el actual contexto de crisis económica y ser un argumento relevante para explicar las diferencias observadas en la eficiencia del Plan E para frenar el aumento del desempleo a escala provincial. ${ }^{7}$

6 Este hecho no se mencionó en ninguna de las evaluaciones realizadas. Sin embargo, si se defendió el criterio de población para el reparto del Fondo por su carácter redistributivo. Dado que la cantidad por habitante es la misma en toda España, pero no lo son ni la renta por habitante, ni la global, se debería producir un mayor impacto en aquellas comunidades y municipios con mayor población y menor renta.

7 Entre las condiciones exigidas a los contratos de obras financiadas con cargo al FEIL, una de ellas fue la inclusión de una cláusula que suponía que el nuevo personal que el contratista necesitase emplear para la ejecución de las obras habría de encontrarse en situación legal de desempleo. De hecho una de las críticas incluidas en el informe del Tribunal de Cuentas (2012) es que en muchos casos las empresas adjudicatarias confundieron la condición de demandante de empleo con la situación legal de desempleo. Por supuesto, a escala provincial es posible evaluar la situación laboral utilizando medidas alternativas de ocupación y paro como las estimadas por la EPA. Sin embargo, como los colectivos incluidos en ambos conceptos de desempleo son diferentes (según los resultados obtenidos por Fernández y Flores (2012) para Galicia solo el 45\% de los parados registrados cumplen con las condiciones para ser considerado parado por la EPA) y dado lo establecido en el Real decreto regulador (el nuevo personal debe encontrase en situación de desempleo) se ha optado por utilizar las cifras administrativas de desempleo, lo que además permitirá comparar estos resultados con los obtenidos para mayores desagregaciones territoriales (municipales o comarcales), lo que no sería posible a partir de las estimaciones que realiza la EPA. Para un análisis detallado de las diferencias entre ambos conceptos de desempleo también puede consultarse Pérez-Infante (2008 y 2009). 


\section{Gráfico 1: Financiación por desempleado (euros)}

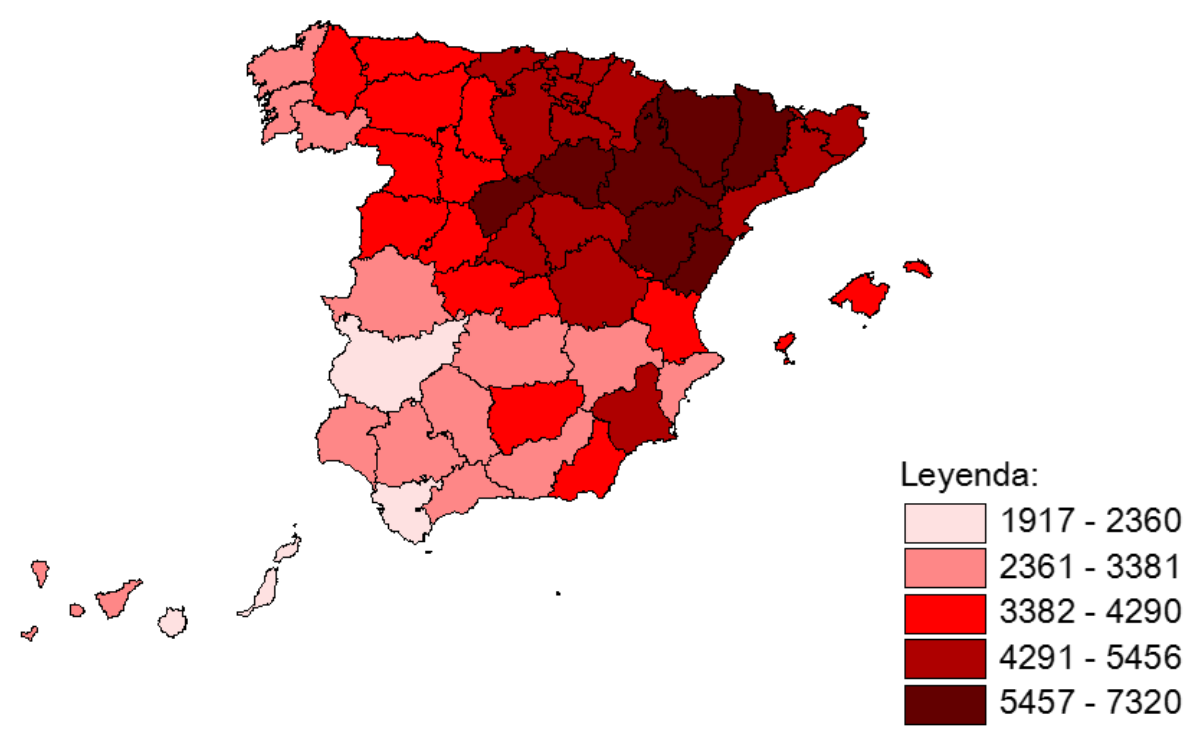

Fuente: Elaboración propia a partir de datos del SISPE y el Informe de seguimiento del FEIL, MPT.

La distribución territorial de la financiación por persona desempleada muestra grandes diferencias territoriales. Mientras se superan los 4.000 euros por desempleado en la zona Nororiental de España, alcanzando los máximos en provincias como Soria, Lleida o Huesca, en el Sudoeste (donde existe un mayor número de parados registrados por habitante), se observan valores que no superan los 2.500 euros por desempleado, con provincias como Cádiz o Ceuta que no alcanzan los 2.000 euros por desempleado (por lo tanto, entre tres y cuatro veces menos que las que más reciben). 
8 Carrascal Incera et al. /Revista de Economía Laboral 11 (2014), 1-23

Cuadro 2. Principales magnitudes del FEIL.

\begin{tabular}{|c|c|c|c|c|c|c|}
\hline Provincia & $\begin{array}{l}\text { Presupuesto } \\
\text { total provincia }\end{array}$ & $\begin{array}{c}\mathrm{N}^{0} \text { de } \\
\text { proyectos }\end{array}$ & $\begin{array}{c}\text { Empleos } \\
\text { presentados }\end{array}$ & $\begin{array}{c}\text { Presupuesto medio } \\
\text { por proyecto } \\
\text { presentado }\end{array}$ & $\begin{array}{l}\text { Empleos } \\
\text { por } \\
\text { proyecto }\end{array}$ & $\begin{array}{c}\text { Multiplicador } \\
\text { directo del } \\
\text { empleo } \\
\end{array}$ \\
\hline Álava & 54.062 .660 & 153 & 1.379 & 353.311 & 9,01 & 25,51 \\
\hline Alicante & 323.050 .311 & 924 & 10.740 & 349.585 & 11,62 & 33,25 \\
\hline Almería & 114.446 .453 & 377 & 3.066 & 303.550 & 8,13 & 26,79 \\
\hline Ávila & 29.846 .948 & 456 & 2.335 & 65.405 & 5,12 & 78,29 \\
\hline Barcelona & 943.792 .208 & 2.324 & 28.629 & 391.698 & 12,32 & 31,45 \\
\hline Burgos & 64.772 .752 & 510 & 2.448 & 127.003 & 4,80 & 37,79 \\
\hline Cáceres & 72.836 .162 & 726 & 4.776 & 100.238 & 6,58 & 65,63 \\
\hline Cádiz & 213.685 .543 & 813 & 7.636 & 254.350 & 9,39 & 36,93 \\
\hline Castellón & 101.464 .186 & 341 & 3.333 & 297.469 & 9,77 & 32,86 \\
\hline Ciudad Real & 90.285 .609 & 529 & 3.766 & 170.667 & 7,12 & 41,71 \\
\hline Granada & 156.475 .145 & 868 & 8.468 & 180.450 & 9,76 & 54,06 \\
\hline Guadalajara & 39.658 .826 & 506 & 2.286 & 78.370 & 4,52 & 57,65 \\
\hline Guipúzcoa & 122.996 .932 & 326 & 2.910 & 377.161 & 8,93 & 23,67 \\
\hline Huelva & 88.081 .929 & 361 & 3.180 & 239.538 & 8,81 & 36,77 \\
\hline Huesca & 38.956 .360 & 402 & 2.105 & 96.610 & 5,24 & 54,20 \\
\hline Jaén & 117.651 .535 & 565 & 6.249 & 208.230 & 11,06 & 53,12 \\
\hline León & 88.031 .666 & 600 & 4.374 & 146.710 & 7,29 & 49,69 \\
\hline Lleida & 73.275 .793 & 436 & 2.523 & 167.949 & 5,79 & 34,46 \\
\hline La rioja & 54.683 .712 & 284 & 2.111 & 192.510 & 7,43 & 38,61 \\
\hline Lugo & 62.861 .986 & 465 & 3.352 & 135.176 & 7,21 & 53,33 \\
\hline Madrid & 1.076 .387 .568 & 1.242 & 23.083 & 866.429 & 18,59 & 21,45 \\
\hline Málaga & 268.583 .764 & 762 & 9.185 & 349.625 & 12,05 & 34,48 \\
\hline Tenerife & 174.124 .595 & 518 & 5.923 & 335.833 & 11,43 & 34,05 \\
\hline Cantabria & 101.383 .123 & 505 & 3.877 & 200.734 & 7,68 & 38,25 \\
\hline Segovia & 28.198 .125 & 353 & 1.661 & 79.870 & 4,71 & 58,91 \\
\hline Sevilla & 327.298 .729 & 1.113 & 12.986 & 294.066 & 11,67 & 39,68 \\
\hline Soria & 16.564 .871 & 260 & 1.119 & 63.704 & 4,30 & 67,56 \\
\hline Tarragona & 134.120 .821 & 509 & 4.435 & 263.482 & 8,71 & 33,07 \\
\hline Teruel & 25.494 .452 & 328 & 1.091 & 77.721 & 3,33 & 42,80 \\
\hline Toledo & 113.205 .401 & 709 & 5.878 & 159.482 & 8,29 & 51,98 \\
\hline Valencia & 440.078 .311 & 1.273 & 13.953 & 345.685 & 10,96 & 31,71 \\
\hline Valladolid & 92.327 .875 & 443 & 3.653 & 208.408 & 8,25 & 39,57 \\
\hline Vizcaya & 202.024 .490 & 412 & 4.168 & 490.233 & 10,12 & 20,64 \\
\hline Zamora & 34.908 .637 & 459 & 2.425 & 76.046 & 5,28 & 69,47 \\
\hline Zaragoza & 165.041 .908 & 686 & 4.844 & 240.574 & 7,06 & 29,35 \\
\hline Ceuta & 13.557 .832 & 34 & 306 & 394.416 & 9,00 & 22,82 \\
\hline Melilla & 12.290 .065 & 21 & 259 & 585.197 & 12,33 & 21,08 \\
\hline España & 8.000 .000 .000 & 30.694 & 277.535 & 259.148 & 9,04 & 34,89 \\
\hline
\end{tabular}

Fuente: Elaboración propia a partir de datos del Informe de seguimiento del FEIL, MPT. 
Las diferencias entre provincias no se reducen a la ratio de financiación por desempleado. Como muestra el Cuadro 2 son también muy importantes las diferencias en la dimensión de los proyectos (el presupuesto medio presentado, varía desde los 866.000 euros por proyecto de Madrid a los 63.000 euros de Soria); en el número de empleos por proyecto (como cabría esperar, aquellas provincias que presentan presupuestos más bajos generan menos empleos por proyecto); o en el multiplicador directo del empleo. ${ }^{8}$ Los datos muestran que aquellas provincias con proyectos de menor envergadura (y consecuentemente menor plazo de ejecución) presentan una capacidad de generación de empleo (número de contratos) mayor que aquellas con proyectos de presupuestos más grandes (el coeficiente de correlación entre tamaño del proyecto y creación de empleo es de -0,77). No obstante, este resultado puede ser consecuencia de la no consideración de la duración temporal del empleo generado o mantenido. Un empleo de seis meses en un proyecto de mayor envergadura si lo ponderamos por su duración es igual a seis empleos de un mes generados por proyectos más modestos, por eso obtenemos la correlación negativa indicada entre tamaño del proyecto y capacidad de generar empleo. ${ }^{9}$

\subsection{El sector de la construcción como principal foco de acción}

Como señalábamos anteriormente, si analizamos con detalle la tipología por obra de los proyectos presentados ${ }^{10}$ (Cuadro 3), la mayoría de estos se corresponden con actividades realizadas por el sector de la construcción. Así, un 31,79\% de los proyectos están destinados a la

8 Definimos el multiplicador directo del empleo, como los empleos necesarios directamente para poder llevar a cabo un proyecto de estas características presupuestado en 1 millón de euros, es decir el número de empleos creados por cada millón de euros.

${ }^{9}$ No considerar la duración del empleo incluido en el proyecto financiado por el FEIL, es uno de los argumentos que explican la enorme diferencia entre la suma de empleos/contratos indicados por los proyectos individuales y los resultados agregados.

10 Como se puede observar, el número total de proyectos financiados por tipo de obra no se corresponde con la cifra total de proyectos aprobados, pues en un mismo proyecto se pueden incluir dos o más tipos de obras. Aunque la mayoría de los proyectos financiados están relacionados con actividades relacionas con la construcción, con poca variabilidad entre provincias, podría ser interesante contar con información desagregada por tipo de proyecto para contrastar si existen diferencias en la efectividad del fondo relacionadas con la tipología de proyectos dominante en cada provincia. 
rehabilitación y mejora de espacios públicos, un 28,89\% a infraestructuras de servicios básicos, un $17,47 \%$ a edificios culturales, educativos 0 deportivos, etc. Llegando a sumar entre los relacionados directamente con la actividad de construcción el 83,03\%. Es lógico pensar, por lo tanto, que la estructura productiva de las provincias podría afectar de alguna manera a los resultados obtenidos por el Plan E. Es decir, aquellas provincias que dependan más del sector de la construcción deberían ver mitigado el efecto de la crisis, al menos en parte, gracias al gasto fiscal realizado.

\section{Cuadro 3: Tipos de proyectos según objetivo del mismo}

\begin{tabular}{lrr}
\hline Tipos de proyectos & $\begin{array}{r}\mathrm{N}^{\mathrm{o}} \text { de } \\
\text { proyectos }\end{array}$ & $\begin{array}{r}\text { Porcentaje } \\
(\%)\end{array}$ \\
\hline Rehabilitación y mejora de espacios públicos & 10.486 & $31,79 \%$ \\
Equipamientos e infraestructuras de servicios básicos & 9.528 & $28,89 \%$ \\
Equipamientos y edificios culturales, educativos o deportivos & 5.762 & $17,47 \%$ \\
Edificios y equipamientos sociales, sanitarios y funerarios & 2.773 & $8,41 \%$ \\
Promoción de la movilidad y seguridad vial & 1.197 & $3,63 \%$ \\
Conservación del patrimonio histórico y municipal & 882 & $2,67 \%$ \\
Supresión de barreras arquitectónicas & 726 & $2,20 \%$ \\
Ahorro y eficiencia energética & 724 & $2,20 \%$ \\
Protección del medio ambiente & 434 & $1,32 \%$ \\
Promoción industrial & 216 & $0,65 \%$ \\
Promoción del turismo & 185 & $0,56 \%$ \\
Prevención de incendios & 69 & $0,21 \%$ \\
\hline Total & 32.982 & $100 \%$ \\
\hline
\end{tabular}

Fuente: Elaboración propia a partir de datos del Informe de seguimiento del FEIL, MPT.

En el Gráfico 2 se clasifican las provincias en función del porcentaje de afiliados en el sector de la construcción sobre el total de la provincia para el año 2007. Cabe destacar que en provincias como Ciudad Real, Ávila, Cáceres, Cuenca o Toledo se supera la cifra del 18\% de los afiliados en la construcción, mientras que en Guipúzcoa, Melilla, Álava o Barcelona no llega a significar el 10\%. Por lo tanto, deberían ser estas provincias, donde el peso de la construcción es más relevante, primero las más sensibles al efecto de la crisis entre 2007 y 2008, y luego entre 2008 y 2009 las que más deberían notar los efectos positivos del esfuerzo inversor público. 
Gráfico 2: Peso del sector de la construcción en el total de afiliados en el año 2007 (en tanto por 1).

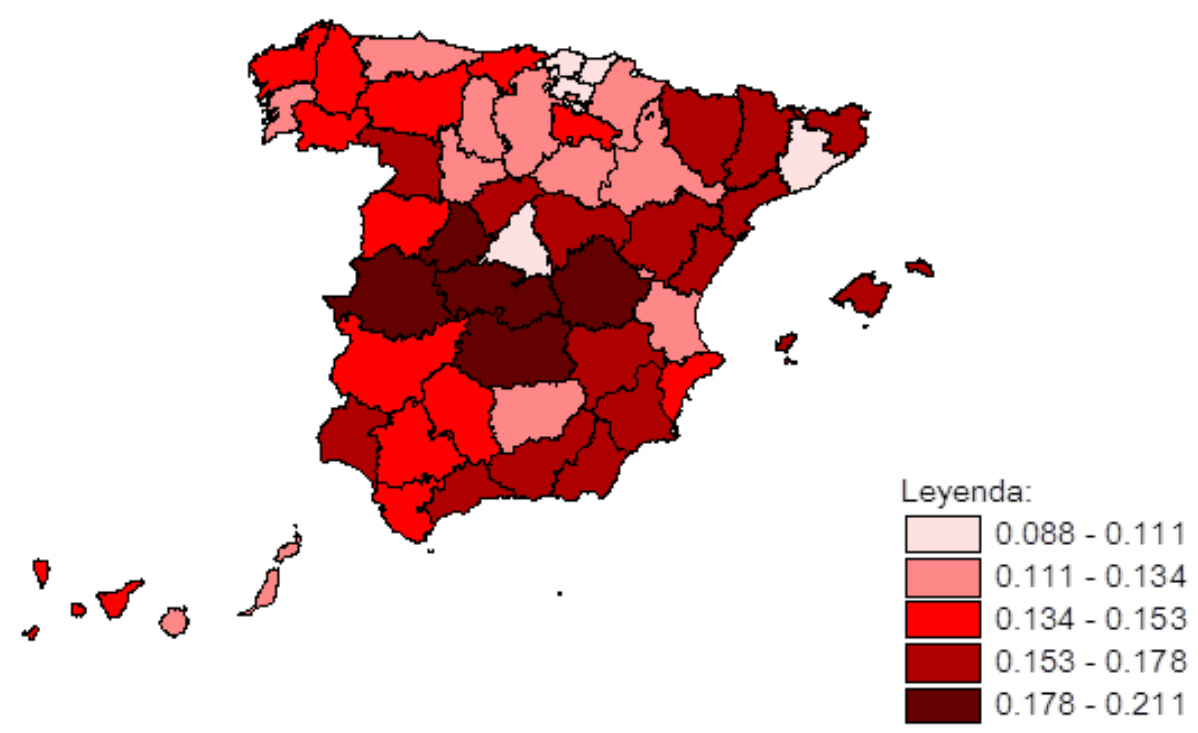

Fuente: Elaboración propia a partir de datos del TGSS.

\subsection{Licitación de obra pública por la Administración central y autonómica por provincia}

A la hora de sacar conclusiones acerca de si las medidas aplicadas mediante el FEIL han sido efectivas o no, en la creación y mantenimiento de empleo, además de otras circunstancias, hay que tener en cuenta la evolución de las fuentes alternativas de financiación de la actividad de construcción civil de las que se nutren las provincias, es decir, la financiación autonómica y la central. La omisión de estas variables en el análisis haría que los resultados del Plan $\mathrm{E}$ sólo fuesen reales en un contexto ceteris paribus. La realidad es otra, la licitación de obra pública de la Administración central y autonómica, varía a lo largo del periodo de 
estudio, y lo hace de tal forma que podemos intuir que lo que se ha dado, en muchos casos, es un efecto sustitución de inversiones.

El Cuadro 4 muestra tanto en valores absolutos como en tasas de variación la inversión central y autonómica por provincias así como la tasa de variación de la inversión local dentro de la que se incluye el FEIL. Los datos confirman que el $67 \%$ de las provincias ven reducida su inversión total (excluida la local) mientras el $84 \%$ de las provincias ha visto aumentados sus fondos locales para inversiones (donde se incluye el FEIL).

La pauta general es que en las provincias en las que aumenta la financiación local se ha reducido la inversión autonómica y central. En el caso contrario, es decir, las provincias en las cuales la inversión local no aumenta, sino que se reduce, la inversión supralocal funciona en sentido inverso. El efecto sustitución de la inversión también está presente a nivel agregado, como puede verse para el total de España, en el que los fondos locales han aumentado un $36 \%$, mientras que los autonómicos y centrales se han reducido en cerca de un $18 \%$. Ahora bien, hay que tener en cuenta las diferencias de magnitud. Es decir, si calculamos la tasa de variación de la inversión agregada veríamos que el incremento de los fondos locales no compensa la caída de los fondos restantes invertidos a nivel local, esta tasa es del $-3,11 \%$ (en valores absolutos la caída es superior a los 1.254 millones de euros).

Algunos ejemplos de provincias en las que se produce un efecto de sustitución de inversiones total o parcial son Alicante, Barcelona, Burgos o A Coruña. En el caso contrario, en los que la bajada de inversión local es compensada con el incremento de la autonómica (sobre todo) y la central, se encuentran provincias como Guipúzcoa, La Rioja o Lugo. Resumiendo, un $23 \%$ de las provincias mejoran el nivel de inversión en obra pública, ya que tanto su inversión local como central y autonómica se han visto incrementadas (Cáceres, Ourense, Palencia, etc.). Mientras, y como casos paradójicos y extremos, Castellón, Málaga y Zaragoza ofrecen tasas de variación negativas para ambos tipos de inversión pública. 


\section{Cuadro 4: Licitación obra pública por provincias (miles de euros)}

\begin{tabular}{|c|c|c|c|c|c|c|c|c|}
\hline \multirow[b]{2}{*}{ Provincia } & \multicolumn{3}{|c|}{ Licitación obra Pública 2009} & \multicolumn{3}{|c|}{ Licitación obra Pública 2007} & \multicolumn{2}{|c|}{ Tasa de variación 09/07 } \\
\hline & Central & Autonómica & $\begin{array}{c}\text { Total } \\
\text { (Central y } \\
\text { Autonómica) }\end{array}$ & Central & Autonómica & $\begin{array}{c}\text { Total } \\
\text { (Central y } \\
\text { Autonómica) }\end{array}$ & $\begin{array}{c}\text { Total } \\
\text { (Central y } \\
\text { Autonómica) }\end{array}$ & $\begin{array}{c}\text { Local } \\
\text { (Plan E } \\
\text { incl.) }\end{array}$ \\
\hline Álava & 125.035 & 89.990 & 215.025 & 19.739 & 144.492 & 164.230 & $30,93 \%$ & $17,36 \%$ \\
\hline Albacete & 157.589 & 81.450 & 239.039 & 208.010 & 40.981 & 248.991 & $-4,00 \%$ & $43,19 \%$ \\
\hline Alicante & 420.452 & 145.931 & 566.383 & 316.523 & 535.577 & 852.100 & $-33,53 \%$ & $101,35 \%$ \\
\hline Almería & 325.400 & 182.996 & 508.397 & 63.696 & 206.472 & 270.168 & $88,18 \%$ & $33,43 \%$ \\
\hline Ávila & 21.431 & 83.450 & 104.881 & 14.331 & 74.698 & 89.029 & $17,81 \%$ & $83,28 \%$ \\
\hline Badajoz & 136.765 & 241.914 & 378.679 & 282.375 & 135.974 & 418.349 & $-9,48 \%$ & $117,85 \%$ \\
\hline Illes Balears & 205.925 & 187.912 & 393.838 & 239.423 & 174.056 & 413.479 & $-4,75 \%$ & $125,82 \%$ \\
\hline Barcelona & 1.465 .349 & 1.210 .949 & 2.676 .298 & 1.617 .966 & 1.938 .583 & 3.556 .549 & $-24,75 \%$ & $65,07 \%$ \\
\hline Burgos & 179.527 & 162.698 & 342.225 & 289.965 & 151.954 & 441.919 & $-22,56 \%$ & $33,88 \%$ \\
\hline Cáceres & 386.905 & 140.154 & 527.060 & 206.436 & 205.849 & 412.285 & $27,84 \%$ & $478,06 \%$ \\
\hline Cádiz & 165.864 & 221.349 & 387.212 & 201.926 & 526.891 & 728.817 & $-46,87 \%$ & $133,14 \%$ \\
\hline Castellón & 145.647 & 151.122 & 296.769 & 89.190 & 240.447 & 329.638 & $-9,97 \%$ & $-53,92 \%$ \\
\hline Ciudad Real & 130.075 & 99.582 & 229.656 & 255.965 & 171.685 & 427.650 & $-46,30 \%$ & $51,71 \%$ \\
\hline Córdoba & 36.904 & 159.885 & 196.789 & 202.288 & 119.509 & 321.797 & $-38,85 \%$ & $132,11 \%$ \\
\hline A Coruña & 492.405 & 203.293 & 695.697 & 546.775 & 305.311 & 852.087 & $-18,35 \%$ & $88,44 \%$ \\
\hline Cuenca & 49.715 & 112.471 & 162.185 & 98.011 & 93.543 & 191.554 & $-15,33 \%$ & $16,09 \%$ \\
\hline Girona & 78.926 & 237.008 & 315.934 & 855.594 & 238.329 & 1.093 .922 & $-71,12 \%$ & $26,16 \%$ \\
\hline Granada & 163.610 & 379.996 & 543.607 & 479.614 & 336.237 & 815.851 & $-33,37 \%$ & $194,54 \%$ \\
\hline Guadalajara & 31.674 & 74.375 & 106.049 & 75.676 & 51.328 & 127.004 & $-16,50 \%$ & $90,64 \%$ \\
\hline Guipúzcoa & 69.823 & 449.956 & 519.779 & 22.523 & 193.632 & 216.155 & $140,47 \%$ & $-43,86 \%$ \\
\hline Huelva & 140.062 & 146.786 & 286.848 & 93.228 & 202.312 & 295.540 & $-2,94 \%$ & $282,48 \%$ \\
\hline Huesca & 112.036 & 122.442 & 234.478 & 219.829 & 44.769 & 264.598 & $-11,38 \%$ & $53,41 \%$ \\
\hline Jaén & 26.373 & 183.114 & 209.487 & 380.626 & 202.346 & 582.972 & $-64,07 \%$ & $245,97 \%$ \\
\hline León & 385.803 & 168.293 & 554.097 & 333.960 & 147.066 & 481.026 & $15,19 \%$ & $54,91 \%$ \\
\hline Lleida & 217.295 & 124.147 & 341.442 & 89.963 & 143.432 & 233.395 & $46,29 \%$ & $15,65 \%$ \\
\hline La Rioja & 178.396 & 148.170 & 326.566 & 66.784 & 46.635 & 113.419 & $187,93 \%$ & $-34,26 \%$ \\
\hline Lugo & 331.106 & 171.851 & 502.957 & 227.289 & 193.022 & 420.311 & $19,66 \%$ & $-11,21 \%$ \\
\hline Madrid & 1.034 .230 & 1.453 .484 & 2.487 .714 & 908.197 & 1.116 .097 & 2.024 .294 & $22,89 \%$ & $37,95 \%$ \\
\hline Málaga & 555.345 & 228.557 & 783.902 & 899.264 & 294.919 & 1.194 .183 & $-34,36 \%$ & $-5,17 \%$ \\
\hline Murcia & 82.793 & 329.495 & 412.288 & 745.895 & 299.995 & 1.045 .890 & $-60,58 \%$ & $32,13 \%$ \\
\hline Navarra & 176.260 & 417.687 & 593.947 & 34.514 & 290.590 & 325.105 & $82,69 \%$ & $-12,95 \%$ \\
\hline Ourense & 378.156 & 45.506 & 423.662 & 59.114 & 121.019 & 180.133 & $135,19 \%$ & $163,99 \%$ \\
\hline Asturias & 123.185 & 413.094 & 536.279 & 513.722 & 409.647 & 923.368 & $-41,92 \%$ & $107,80 \%$ \\
\hline Palencia & 528.880 & 87.209 & 616.089 & 57.880 & 112.386 & 170.265 & $261,84 \%$ & $31,08 \%$ \\
\hline Las Palmas & 87.362 & 136.379 & 223.740 & 233.659 & 359.640 & 593.299 & $-62,29 \%$ & $109,85 \%$ \\
\hline Pontevedra & 356.347 & 100.553 & 456.900 & 307.674 & 212.765 & 520.439 & $-12,21 \%$ & $121,69 \%$ \\
\hline Salamanca & 24.181 & 113.067 & 137.248 & 74.840 & 111.383 & 186.223 & $-26,30 \%$ & $97,14 \%$ \\
\hline Tenerife & 61.364 & 112.076 & 173.440 & 172.996 & 366.317 & 539.313 & $-67,84 \%$ & $59,73 \%$ \\
\hline Cantabria & 102.227 & 186.865 & 289.091 & 164.616 & 145.095 & 309.711 & $-6,66 \%$ & $251,08 \%$ \\
\hline Segovia & 43.500 & 43.480 & 86.980 & 24.156 & 46.348 & 70.504 & $23,37 \%$ & $15,21 \%$ \\
\hline Sevilla & 169.919 & 828.038 & 997.957 & 469.784 & 603.340 & 1.073 .124 & $-7,00 \%$ & $18,23 \%$ \\
\hline Soria & 175.309 & 133.753 & 309.061 & 286.241 & 29.632 & 315.873 & $-2,16 \%$ & $92,90 \%$ \\
\hline Tarragona & 413.094 & 217.224 & 630.318 & 522.899 & 229.612 & 752.511 & $-16,24 \%$ & $9,82 \%$ \\
\hline Teruel & 56.077 & 60.541 & 116.618 & 85.852 & 28.231 & 114.083 & $2,22 \%$ & $-27,96 \%$ \\
\hline Toledo & 115.779 & 139.717 & 255.497 & 474.517 & 216.740 & 691.257 & $-63,04 \%$ & $35,98 \%$ \\
\hline Valencia & 660.167 & 354.101 & 1.014 .268 & 1.225 .361 & 741.547 & 1.966 .908 & $-48,43 \%$ & $5,94 \%$ \\
\hline Valladolid & 50.083 & 250.072 & 300.155 & 484.230 & 89.959 & 574.189 & $-47,73 \%$ & $124,46 \%$ \\
\hline Vizcaya & 84.617 & 306.830 & 391.448 & 556.011 & 235.434 & 791.445 & $-50,54 \%$ & $13,16 \%$ \\
\hline Zamora & 466.072 & 95.377 & 561.448 & 180.281 & 60.838 & 241.120 & $132,85 \%$ & $87,95 \%$ \\
\hline Zaragoza & 137.409 & 165.752 & 303.161 & 192.768 & 185.907 & 378.675 & $-19,94 \%$ & $-28,89 \%$ \\
\hline Ceuta & 148.564 & 24.644 & 173.209 & 28.313 & 18.928 & 47.241 & $266,65 \%$ & $53,91 \%$ \\
\hline Melilla & 9.411 & 37.997 & 47.408 & 82.294 & 13.184 & 95.478 & $-50,35 \%$ & s.d. \\
\hline España & 12.220 .420 & 11.962 .783 & 24.183 .203 & 16.282 .784 & 13.204 .683 & 29.487 .467 & $-17,99 \%$ & $36,36 \%$ \\
\hline
\end{tabular}




\section{Evaluación de la efectividad en la creación y mantenimiento de empleo del Plan E}

Según el informe del MPT, las obras ejecutadas durante 2009 habrían dado empleo a 426.195 personas cifra muy superior a las 277.539 inicialmente previstas, de la cuales 181.335 son de nueva contratación y 244.860 ya estaban contratadas en las empresas adjudicatarias y mantienen, por tanto, su empleo. Como entre las condiciones exigidas a las obras financiadas por el FEIL, se incluía una cláusula que suponía que el nuevo personal habría de encontrarse en situación legal de desempleo, parece más apropiado analizar el impacto sobre el empleo del FEIL utilizando como medida de referencia las variaciones en el número de desempleados registrados en las oficinas del servicio público de empleo pero teniendo en cuenta cual es la situación de partida. Por ello hemos utilizado como variable de evaluación de los efectos del Plan E sobre el empleo una Ratio de Ajuste Territorial del Mercado Laboral (RATML) ${ }^{11}$ que definimos como:

$$
R A T M L_{i t}=\left(1-\frac{\text { Parados registrados }_{i t}}{\text { Afiliados }_{i t}}\right), \quad \forall i \in\{1, \ldots, n\}
$$

Siendo $i$ el número de territorios y $t$ el año de estudio.

La ratio trata de aproximar la capacidad que tiene un territorio para generar empleo para sus residentes, ya que los parados registrados lo son en su lugar de residencia mientras que los datos de afiliación están recogidos por centro de cotización. Valores de la ratio próximos a cero implican que el volumen de parados para esa provincia es similar al número de afiliados, lo que significa una situación muy negativa del mercado de trabajo; por el contrario, una ratio elevada y próxima a uno implica un cociente entre parados y afiliados bajo por lo que la situación de la provincia es buena laboralmente, es decir, es capaz de generar empleo para el montante de población activa local. A través de la evolución de esta ratio

11 Esta ratio ha sido empleada con anterioridad en un estudio realizado por la Cámara de Comercio de Cáceres (2005) en el que, bajo la denominación de ratio de eficiencia, se utiliza esta relación para comparar el comportamiento de los mercados de trabajo a nivel municipal. 
sabremos si el agregado de mercados locales delimitados por la provincia ha respondido de forma positiva al estímulo fiscal definido por el FEIL. De cara al análisis posterior del RATML se ha determinado que valores superiores a 0,8 reflejaran una situación de buen ajuste laboral a nivel territorial. Esto implica una relación mínima de cinco afiliados por cada parado registrado.

En el año 2007 (Cuadro 5) la mayoría de las provincias tienen ratio superior o cercano al 0,80, lo que reflejaría una buena situación de partida. Desde este año la crisis económica ha generado un empeoramiento del ajuste entre oferta y demanda de trabajo presentando todas las provincias una caída en el RATML en 2009. Para el conjunto de España el descenso en el indicador es de un 13,6\% pasando de un mercado laboral con un elevado ajuste $(0,88)$ a uno sensiblemente menos solvente $(0,76)$. La caída es muy considerable a escala local con un $67,3 \%$ de las provincias con RATML inferiores a 0,80 frente al $11,5 \%$ que se encontraban en esa situación en 2007.

Más concretamente, los mercados más eficientes en el 2007 serían los de Zaragoza, Lleida, Castellón o Álava (todos ellos por encima del 0,93). Por el lado contrario, destacan tanto Ceuta y Melilla, como Badajoz, Cádiz o Córdoba. En 2009, teniendo en cuenta el efecto de la crisis, el mejor ajuste se obtiene en Euskadi, Navarra, Madrid o Soria. El peor ajuste lo ofrecen la mayor parte de las provincias Andaluzas, las Islas Canarias, Alicante y, como ya sucedía en 2007, Ceuta y Melilla.

En términos relativos, las caídas más pronunciadas en el RATML en este período (Gráfico 3) las encontramos en Castellón, Toledo, Alicante y las provincias de Andalucía y las Islas Canarias (alcanzando el máximo en Cádiz con una caída del 32,6\%, del 0,69 al 0,46). Las que mejor reaccionan a la crisis son las provincias del Norte, es decir, las de Galicia, el País Vasco o Asturias. 


\section{Cuadro 5: Evolución de la Ratio de Ajuste Territorial del Mercado} Laboral 2009-2007

\begin{tabular}{|c|c|c|c|}
\hline Provincia & RATML 2009 & RATML 2007 & Tv 09/07 \\
\hline Álava & 0,8709 & 0,9315 & $-6,5069 \%$ \\
\hline Albacete & 0,6996 & 0,8292 & $-15,6356 \%$ \\
\hline Alicante & 0,6294 & 0,8469 & $-25,6776 \%$ \\
\hline Almería & 0,7054 & 0,8649 & $-18,4447 \%$ \\
\hline Ávila & 0,7518 & 0,8630 & $-12,8933 \%$ \\
\hline Badajoz & 0,6235 & 0,7410 & $-15,8612 \%$ \\
\hline Illes Balears & 0,7425 & 0,8732 & $-14,9717 \%$ \\
\hline Barcelona & 0,8163 & 0,9197 & $-11,2447 \%$ \\
\hline Burgos & 0,8290 & 0,9146 & $-9,3636 \%$ \\
\hline Cáceres & 0,6908 & 0,8068 & $-14,3855 \%$ \\
\hline Cádiz & 0,4648 & 0,6903 & $-32,6626 \%$ \\
\hline Castellón & 0,7313 & 0,9301 & $-21,3691 \%$ \\
\hline Ciudad Real & 0,6831 & 0,8384 & $-18,5248 \%$ \\
\hline Córdoba & 0,6528 & 0,7834 & $-16,6604 \%$ \\
\hline A Coruña & 0,7799 & 0,8482 & $-8,0521 \%$ \\
\hline Cuenca & 0,7754 & 0,8779 & $-11,6772 \%$ \\
\hline Girona & 0,8035 & 0,9189 & $-12,5606 \%$ \\
\hline Granada & 0,6571 & 0,8265 & $-20,5029 \%$ \\
\hline Guadalajara & 0,7674 & 0,9188 & $-16,4749 \%$ \\
\hline Guipúzcoa & 0,8725 & 0,9267 & $-5,8484 \%$ \\
\hline Huelva & 0,6233 & 0,7854 & $-20,6438 \%$ \\
\hline Huesca & 0,8599 & 0,9350 & $-8,0331 \%$ \\
\hline Jaén & 0,7366 & 0,8298 & $-11,2319 \%$ \\
\hline León & 0,7774 & 0,8556 & $-9,1301 \%$ \\
\hline Lleida & 0,8530 & 0,9444 & $-9,6763 \%$ \\
\hline La rioja & 0,8138 & 0,9128 & $-10,8449 \%$ \\
\hline Lugo & 0,8251 & 0,8559 & $-3,5913 \%$ \\
\hline Madrid & 0,8355 & 0,9230 & $-9,4722 \%$ \\
\hline Málaga & 0,6382 & 0,8240 & $-22,5464 \%$ \\
\hline Murcia & 0,7286 & 0,9003 & $-19,0763 \%$ \\
\hline Navarra & 0,8408 & 0,9193 & $-8,5382 \%$ \\
\hline Ourense & 0,7598 & 0,8112 & $-6,3378 \%$ \\
\hline Asturias & 0,7953 & 0,8641 & $-7,9665 \%$ \\
\hline Palencia & 0,8091 & 0,8814 & $-8,2033 \%$ \\
\hline Las Palmas & 0,6144 & 0,8063 & $-23,8031 \%$ \\
\hline Pontevedra & 0,7340 & 0,8369 & $-12,2983 \%$ \\
\hline Salamanca & 0,7614 & 0,8503 & $-10,4554 \%$ \\
\hline Sta Cruz de Tenerife & 0,6428 & 0,8332 & $-22,8475 \%$ \\
\hline Cantabria & 0,7952 & 0,8987 & $-11,5154 \%$ \\
\hline Segovia & 0,8426 & 0,9232 & $-8,7346 \%$ \\
\hline Sevilla & 0,6562 & 0,8162 & $-19,6054 \%$ \\
\hline Soria & 0,8733 & 0,9362 & $-6,7188 \%$ \\
\hline Tarragona & 0,7757 & 0,9042 & $-14,2026 \%$ \\
\hline Teruel & 0,8453 & 0,9241 & $-8,5227 \%$ \\
\hline Toledo & 0,7030 & 0,8813 & $-20,2293 \%$ \\
\hline Valencia & 0,7428 & 0,8992 & $-17,3932 \%$ \\
\hline Valladolid & 0,7974 & 0,8827 & $-9,6550 \%$ \\
\hline Vizcaya & 0,8463 & 0,9137 & $-7,3793 \%$ \\
\hline Zamora & 0,7655 & 0,8366 & $-8,4949 \%$ \\
\hline Zaragoza & 0,8252 & 0,9317 & $-11,4295 \%$ \\
\hline Ceuta & 0,5789 & 0,6464 & $-10,4326 \%$ \\
\hline Melilla & 0,5527 & 0,6434 & $-14,0922 \%$ \\
\hline España & 0,7612 & 0,8809 & $-13,5835 \%$ \\
\hline
\end{tabular}

Fuente: Elaboración propia. 


\section{Gráfico 3: Variación relativa de la Ratio de Ajuste Territorial del Mercado Laboral 2009-2007}

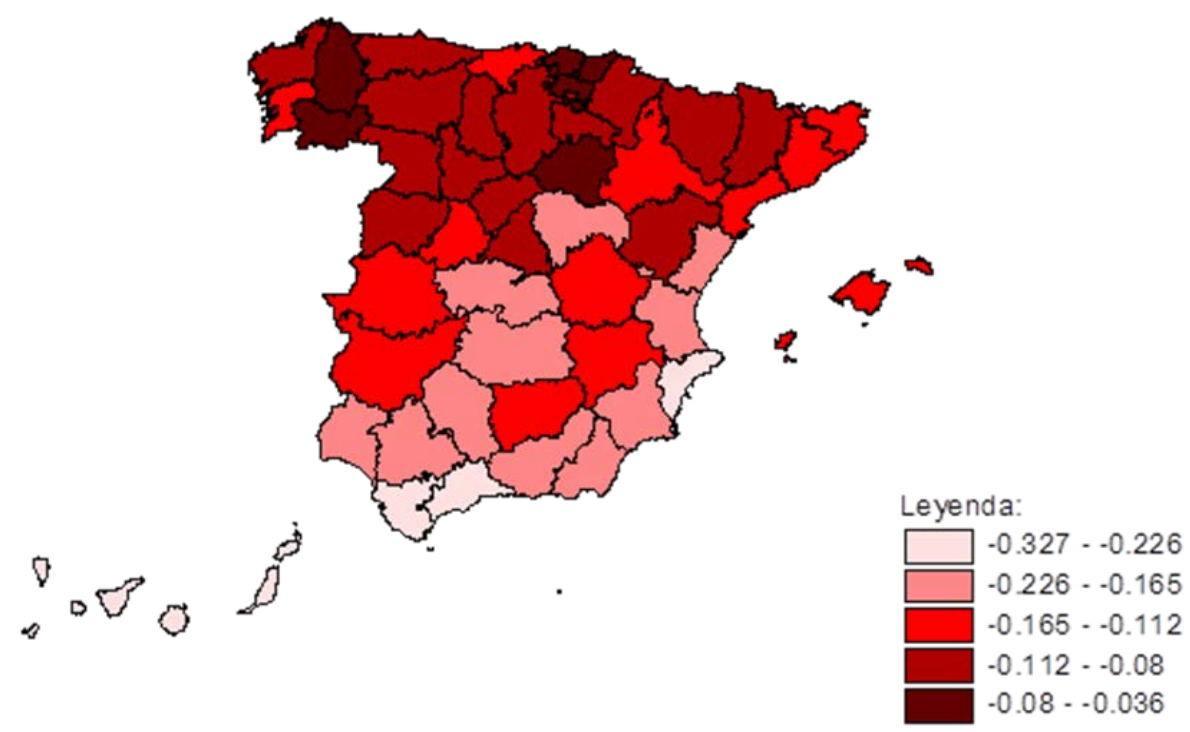

\subsection{Efectos del FEIL sobre la evolución del mercado laboral provincial}

A continuación presentamos un modelo estructural construido ad hoc con el objetivo de analizar los efectos sobre el mercado laboral a escala provincial de las inversiones asociadas al Plan E durante el año 200912. El objetivo es ofrecer evidencia empírica que apoye las afirmaciones y conclusiones realizadas. La hipótesis de partida consiste en vincular la evolución del RATML durante el periodo de análisis a la evolución de las variables que se han presentado de forma descriptiva en las secciones anteriores. Teniendo en cuenta el objetivo principal del FEIL y sus cláusulas de empleo, los efectos de estos fondos sobre la evolución del

12 A fecha 1 de julio de 2010, el 93,6\% de los proyectos estaban ya totalmente liquidados. 
mercado laboral deberán ser claramente positivos, al incidir directamente sobre el número de parados registrados. Para poder contrastar esta hipótesis se han estimado dos modelos en los que se hace depender la evolución del RATML de la financiación del Plan E (sin ponderar o ponderado por el número de desempleados), el peso de la afiliación en el sector de la construcción en el total de afiliados (AFCONST), la variación en la licitación pública (central y autonómica) en la provincia, excluidos por tanto los fondos del FEIL (TVINVPUB) y la tasa de crecimiento real de la producción provincial entre 2007 y 2009 (TVPIB). Es decir:

$$
\Delta \text { RATML }(09-07)=\mathrm{f}(\text { FEIL; AFCONST; TVINVPUB; TVPIB })
$$

Como se mostró en el Cuadro 5, el RATML, que se presenta en diferencias, muestra siempre valores negativos debido a que la situación del mercado laboral español ha empeorado considerablemente durante este periodo (las diferencias se han calculado sustrayendo de la Ratio de 2009 el valor de la misma en 2007). En el primer modelo se ha incluido para cada provincia el valor total del fondo asignado, mientras que en el segundo modelo se pondera por el número de desempleados. De esta forma, podemos contrastar si la incidencia del fondo ha sido o no independiente del número de desempleados existentes en cada provincia.

Los resultados de la estimación mínimo cuadrática ordinaria de los modelos propuestos se presentan en el Cuadro 6. El modelo 1 ofrece unos resultados claramente insatisfactorios, pero permite contrastar que el reparto por población no ha sido efectivo desde el punto de vista laboral. A mayor población el presupuesto del fondo es mayor (proporcional a la población) pero su efecto sobre la evolución del ratio es negativo. Como ya se mostraba en el Cuadro 2, el efecto sobre la contratación es mayor cuanto menor es el tamaño medio de los proyectos. Respecto al modelo 2 , todas las variables explicativas incluidas son significativas a un nivel del $1 \%$, con la excepción del peso de la construcción que lo es al 5\%, y además, este modelo explica el 63,9\% del comportamiento observado en la evolución del RATML provincial. La ausencia de multicolinealidad, comprobada mediante el Test de Farrar-Glauber, confirma que los estimadores del modelo son LIO (Lineales, Insesgados y Óptimos). 


\section{Cuadro 6: Factores determinantes del comportamiento del RATML}

\begin{tabular}{lll}
\hline Variable dependiente: Diferencias en el RATML 2009-2007 & Modelo 1 & Modelo 2 \\
Variables independientes: & & \\
\hline Presupuesto Plan E por provincia & $-0.017^{* * *}$ & \\
Financiación por desempleado & & $0.065^{* * *}$ \\
\% Afiliados construcción (07) & $-0.005^{* * *}$ & $-0.003^{* *}$ \\
Inversión pública restante (07-09) & 0.009 & $0.016^{* * *}$ \\
PIB (07-09) & $0.556^{* * *}$ & $0.789^{* * *}$ \\
Constante & $0.269^{* *}$ & $-0.612^{* * *}$ \\
\hline $\mathrm{N}^{\circ}$ de observaciones & 52 & 52 \\
$\mathrm{R}^{2}$ ijustado & 0.535 & 0.639 \\
$\mathrm{R}^{2}$ ajusta & 0.495 & 0.608 \\
\hline
\end{tabular}

*** Significativo al 1\%, ** Significativo al $5 \%, *$ Significativo al $10 \%$.

La financiación por desempleado incrementa la eficiencia del mercado laboral local, concretamente, un incremento de un punto porcentual en la financiación mejoraría en siete centésimas el diferencial de la ratio; mientras que el peso de la construcción tiene un efecto negativo. En principio no debería de ser así, ya que el 80\% de los puestos de trabajo generados por el Plan E, lo han sido en el Sector de la construcción. Sin embargo, el fuerte efecto negativo sobre el empleo de la crisis inmobiliaria no ha sido compensado por la aplicación de fondos públicos, quizás en gran medida por la propia formula de reparto de los mismos o por la propia falta de seguimiento de los proyectos. La distribución de los fondos en función de la población, ha propiciado que se hayan generado menor cantidad de puestos de trabajo en las provincias donde el sector construcción es más grande (en función de la cantidad de afiliados o desempleados) que en provincias con menor importancia del sector construcción, lo que a escala agregada ha suavizado el efecto positivo del Plan E sobre la generación de empleo. ${ }^{13}$

El coeficiente de la tasa de variación de la Inversión Pública no local (central y autonómica) muestra una relación positiva con el diferencial del RATML. Es decir, el Plan E tiene diferentes efectos en función de cuál es la

13 Cabe destacar que el peso de la construcción en el total de afiliados provinciales tiene una correlación negativa con el tamaño poblacional. Es decir, a mayor población, menor peso de la afiliación del sector construcción en la provincia. Por lo tanto, el reparto en función de la población no ha favorecido a las provincias con mayor dependencia del sector construcción. 
actitud tomada por el resto de la administración pública en cuanto a mantener o no su nivel de actuación (aditividad frente a sustitución). O lo que es lo mismo, con una inversión local dada, a mayor inversión Central y Autonómica se produce una mejora de la eficiencia del mercado laboral como era de esperar. En el modelo propuesto, un incremento unitario en la variación de la inversión genera una mejora de dos centésimas en el diferencial. Por último, la variación del PIB, que nos ayuda a controlar las diferencias provinciales y a mejorar la estimación de los parámetros de las demás variables, presenta correlación positiva con el diferencial de la ratio lo que es indicativo de que aquellas provincias en las que aumenta (disminuye) la producción, mejoran (empeoran) la eficiencia del mercado laboral. En este caso, un incremento de un punto en la variación mejoraría la ratio en cerca de ocho décimas.

\section{Conclusiones}

A través del presente trabajo, se han tratado de evaluar los efectos que el Plan Español para el Estímulo de la Economía y el Empleo ha producido a nivel provincial a través de las medidas contempladas en el Fondo Estatal de Inversión Local (FEIL) en los resultados observados en el mercado laboral local. Mediante las distintas herramientas utilizadas en el análisis, se ha podido constatar la existencia de resultados y comportamientos heterogéneos y se ha profundizado en algunas de las posibles causas de estas divergencias. Se ha podido observar, en primer lugar, como un reparto homogéneo del Fondo (en términos per cápita) puede dar lugar a resultados muy heterogéneos en términos laborales. La evidencia empírica aportada parece confirmar que el ajuste del mercado de trabajo es independiente del tamaño de la población, lo que debería tenerse en consideración para un mejor aprovechamiento de los fondos disponibles. También se ha podido constatar la influencia, sobre el RATML, de la estructura productiva de la provincia y de la evolución agregada de la producción provincial. La relevancia del peso de la afiliación en la construcción para explicar la evolución del RATML permite pensar en alternativas de reparto de los fondos diferente a la realizada teniendo en cuenta no sólo el volumen de parados sino también la estructura sectorial de la provincia. Por último, también es destacable la necesidad de evaluar cada medida de política fiscal considerando el conjunto de medidas adoptadas simultáneamente. La incidencia del FEIL no es independiente de lo que suceda con el conjunto de la licitación pública, como los resultados obtenidos han confirmado. En este sentido, es necesario tener en consideración distintos escenarios en las evaluaciones exante del impacto 
de programas, lo cual puede ser una exigencia para los nuevos proyectos de inversión dentro del horizonte 2020.

El modelo econométrico planteado confirma algunas de las relaciones descritas anteriormente y desmiente otras que, si bien, se presuponían inversas a las finalmente estimadas, dada la naturaleza de los proyectos contemplados al amparo del FEIL, han podido ser matizadas convenientemente. Nos referimos aquí al impacto negativo que produce un mercado laboral muy volcado hacia la construcción sobre la evolución del mismo en este período de crisis. Desde este punto de vista, los efectos del FEIL se han dejado sentir con mayor intensidad en el sector de la construcción (aproximadamente el $80 \%$ de los proyectos tenían como principales ejecutores agentes de este sector), pero no lo han hecho de forma homogénea interprovincialmente ni con la intensidad necesaria, en gran medida por el criterio de reparto elegido por las autoridades públicas.

Existe una correlación positiva entre financiación por desempleado y mejora de la Ratio de Ajuste Territorial de Mercado Laboral, lo cual parece indicar que el reparto de fondos por persona desempleada podría tener unos efectos más beneficiosos en términos de ajuste laboral que un reparto por población. Para mejorar los resultados del FEIL, por lo tanto, la distribución de los fondos se debería haber realizado mediante otro criterio que no fuese estrictamente la población total. Aunque en este modelo queda demostrado que el reparto en función de la población desempleada es mejor que el utilizado, tampoco sería un reparto óptimo. Otra propuesta de reparto podría consistir en un índice compuesto que ponderara las variables recogidas en el modelo, sobre todo, teniendo en cuenta la magnitud del sector construcción, ya que este plan de estímulo estaba encaminado a frenar el varapalo que se estaba produciendo en el mismo debido a la crisis inmobiliaria. Eso sí, habría que tener muy en cuenta los posibles desequilibrios territoriales que, si bien ya existen, podrían verse agravados. Por último, la posible existencia de sustitución de inversión entre las diferentes administraciones con competencias en la financiación de la dotación de capital público puede diluir completamente los efectos positivos de un plan de estímulo como el diseñado. Este hecho parece apoyar la idea de que es necesario tener en cuenta los posibles efectos de escala redistributivos y desbordamiento que pueden provocar modificaciones en la distribución de la inversión pública entre los distintos niveles de la administración, a la hora de realizar el planteamiento de las medidas fiscales de estímulo económico. 


\section{Bibliografía}

BOE (2008). Real Decreto-Ley 9/2008, del 28 de noviembre. Boletín Oficial del Estado, 2 de diciembre, n 290 , p. 48125.

Cámara Oficial de Comercio e Industria de la provincia de Cáceres (2005). Cáceres: Economía Provincial 2005. Datos pueblo a pueblo, $\mathrm{n}^{\circ} 80$. Disponible en:

http://www.camaracaceres.es/actividades/publicaciones/libros/completos/8 0/contenidos/index2.htm. Consulta: 29/11/2011.

Carrascal-Incera, A., Corbelle, F., Fernández, M. y Vilariño, M.C. (2011). "Plan E como estímulo fiscal. Evaluación de la eficiencia a nivel provincial". Encuentro de Economía Pública. Málaga.

Espadas Moncalvillo, L. (2009). "Presupuestos Generales del Estado para 2009: unas cuentas públicas para afrontar la coyuntura", Presupuesto y Gasto Público, 54, 45-59, Secretaría General de Presupuestos y Gastos, Instituto de Estudios Fiscales.

Fernandez, M. y Flores, M. (2012). "Las diferencias entre el paro registrado y el paro EPA en Galicia: una evaluación con microdatos". Revista de Economía Laboral, 9, 46-72.

ICE (2009). "Los presupuestos generales del estado para 2009", Boletín Económico del ICE, 2960, Secretaría General de Presupuestos y Gastos.

INE (2009). Demografía y población, Cifras de población y Censos demográficos. Ministerio de Economía y Hacienda. Gobierno de España.

MPT (2010). Informe de seguimiento del Fondo Estatal de Inversión Local (FEIL). Ministerio de Política Territorial y Administraciones Públicas. Gobierno de España.

Pérez-Infante, J.I. (2008). “Por qué el paro registrado supera al paro de la EPA?" Revista del Ministerio de Trabajo e Inmigración. Economía y Sociología, 71, 53-65.

Pérez-Infante, J.I. (2009). "La medición del paro: el paro de la EPA y el paro registrado", Cuadernos de mercado de trabajo, Observatorio de las Ocupaciones del Servicio Público de Empleo Estatal, 3. 
SEOPAN (2009). Observatorio de la construcción. La Asociación de Empresas Constructoras del Ámbito Nacional (SEOPAN).

SEPE (2009). Estadísticas de paro registrado. Servicio Público de Empleo Estatal. Ministerio de Trabajo e Inmigración. Gobierno de España.

TGSS (2009). Estadísticas de afiliación. Tesorería General de la Seguridad Social. Ministerio de Trabajo e Inmigración. Gobierno de España.

Tribunal de Cuentas (2012). Informe de fiscalización de las contrataciones desarrolladas por las entidades locales en relación con las inversiones financiadas por el Fondo Estatal de Inversión Local creado por el Real Decreto-Ley 9/2008, de 28 de noviembre, 948

Uxó, J., Paúl, J. y Salinas, J. (2010). “Análisis y valoración de las medidas discrecionales de estímulo fiscal aplicadas en España en 2009”, Presupuesto y Gasto Público, 59, 55-82. Disponible en: http://www.ief.es/documentos/recursos/publicaciones/revistas/presu gasto _publico/59-04.pdf. Consulta: 25/02/2014. 